\title{
Uma revisão sistemática abordando o Tangram, o GeoGebra e as opções de isometria do plano
}

\section{A systematic review addressing Tangram, GeoGebra and options of the isometry of the plane}

\section{Una revisión sistemática que aborda el Tangram, GeoGebra y las opciones de isometría del plano}

\author{
Robério Pereira Rocha ${ }^{1}$ \\ Secretaria de Educação do Estado da Bahia \\ http://orcid.org/0000-0002-9729-1849 \\ Maria Deusa Ferreira da Silva ${ }^{2}$ \\ Universidade Estadual do Sudoeste da Bahia (UESB) \\ http://orcid.org/0000-0003-3462-3882
}

\begin{abstract}
Resumo
Este artigo apresenta os resultados de parte uma pesquisa de mestrado, e tem como objetivo mapear e analisar pesquisas publicadas de 2015 até 2020, realizadas em nível de ensino básico sobre a utilização do Tangram, GeoGebra e opções de isometria do plano no ensino da matemática. A metodologia utilizada foi a revisão sistemática de literatura, e partiu de buscas de artigos e dissertações realizadas nos periódicos da Biblioteca Digital Brasileira de Teses e Dissertações (BDTD), no Banco de Teses e Dissertações da CAPES e no Google Acadêmico. Usamos as duplas de descritores GeoGebra/Tangram, Tangram/Matemática e GeoGebra/Isometrias e localizamos 293 pesquisas. Após a seleção, guiada fielmente pelo protocolo estabelecido na revisão sistemática, consideramos dez estudos como amostra final da revisão. Concluímos que os trabalhos analisados apontam para a importância da variação de metodologias e utilização de recursos como GeoGebra e Tangram para potencializar o ensino de matemática. A pesquisa evidenciou a significativa contribuição do GeoGebra como incentivador do interesse dos alunos pelo estudo das isometrias do plano. Além disso,
\end{abstract}

\footnotetext{
${ }^{1}$ roberio.rocha2005@gmail.com

2 maria.deusa@uesb.edu.br
} 
constatamos a importância do Tangram utilizado conjuntamente com o GeoGebra como facilitador da apropriação dos conceitos geométricos, instigador da curiosidade, propulsor da criatividade e mediador da percepção espacial. Todavia, percebemos a existência de uma lacuna nesse rol de estudos. Não encontramos estudos que utilizassem simultaneamente estes três elementos: GeoGebra, Simetria e Tangram. E é nessa nova perspectiva, a partir dessa lacuna, que estamos organizando esta pesquisa, ou seja, à luz da teoria da atividade, queremos investigar as estratégias matemáticas dos alunos envolvidos nessas construções de isometrias, durante a formação das figuras do Tangram no ambiente do GeoGebra.

Palavras-chave: GeoGebra, Ensino básico, Tangram.

\begin{abstract}
This article presents the results of part of a master's research and aims to map and analyse research published from 2015 through 2020 at basic education level on the use of Tangram, GeoGebra and options of isometry of the plane in mathematics teaching. The methodology used was the systematic literature review and started from searches for articles and theses carried out in the journals of the Biblioteca Digital Brasileira de Teses e Dissertações/Brazilian Digital Library of Theses and Dissertations (BDTD), CAPES Banco de Teses e Dissertações/Thesis and Dissertations Bank, and Google Acadêmico/ Google Scholar. We used the pairs of descriptors GeoGebra/Tangram, Tangram/Mathematics and GeoGebra/Isometries and found 293 studies. After the selection, guided faithfully by the protocol established in the systematic review, we considered ten studies as the final sample of the review. We concluded that the works analysed point to the importance of varying methodologies and using resources such as GeoGebra and Tangram to enhance mathematics teaching. The research showed the significant contribution of GeoGebra as an incentive for the student's interest in studying the isometries of the plane. In addition, we note the importance of the Tangram used together with
\end{abstract}


GeoGebra as a facilitator of the appropriation of geometric concepts, an instigator of curiosity, a propeller of creativity, and a mediator of spatial perception. However, we perceived a gap in this list of studies. We did not find studies that used these three elements simultaneously: GeoGebra, Simetria, and Tangram. It is from this new perspective, from this gap, that we are organising this research, i.e., in the light of the theory of activity, we want to investigate the mathematical strategies of the students involved in those isometric constructions, during the formation of the Tangram figures in the environment of the GeoGebra.

Keywords: GeoGebra, Basic education, Tangram

\section{Resumen}

Este artículo presenta los resultados de parte de una investigación de maestría y tiene como objetivo mapear y analizar investigaciones publicadas desde 2015 hasta 2020 en nivel de educación básica sobre el uso de Tangram, GeoGebra y opciones de isometría del plano en la enseñanza de las matemáticas. La metodología utilizada fue la revisión sistemática de la literatura y partió de búsquedas de artículos y tesis realizadas en las revistas de la Biblioteca Digital Brasileira de Teses e Dissertações (BDTD), Banco de Teses e Dissertações de CAPES y Google Académico. Utilizamos los pares de descriptores GeoGebra/Tangram, Tangram/Matemáticas y GeoGebra/Isometrías y encontramos 293 estudios. Tras la selección, guiados fielmente por el protocolo establecido en la revisión sistemática, consideramos diez estudios como muestra final de la revisión. Concluimos que los trabajos analizados apuntan a la importancia de variar metodologías y utilizar recursos como GeoGebra y Tangram para potenciar la enseñanza de las matemáticas. La investigación mostró la importante contribución de GeoGebra como incentivo al interés del estudiante por estudiar las isometrías del plano. Además, notamos la importancia del Tangram utilizado junto con GeoGebra como facilitador de la apropiación de conceptos geométricos, instigador de la curiosidad, propulsor de la creatividad y mediador de la percepción espacial. Sin embargo, percibimos una brecha en esta 
lista de estudios. No encontramos estudios que utilizaran estos tres elementos simultáneamente: GeoGebra, Simetria y Tangram. Es desde esta nueva perspectiva, desde esta brecha, que estamos organizando esta investigación, es decir, a la luz de la teoría de la actividad, queremos investigar las estrategias matemáticas de los estudiantes involucrados en esas construcciones isométricas, durante la formación de las figuras de Tangram en el entorno de GeoGebra.

Palabras clave: GeoGebra, Educación básica, Tangram. 


\section{Uma revisão sistemática abordando o Tangram, o GeoGebra e as opções de isometria do plano}

Este estudo está vinculado aos resultados parciais de uma pesquisa de mestrado, e tem como finalidade mapear, descrever e analisar estudos acadêmicos, publicadas entre os anos de 2015 a 2020, que abordem o GeoGebra ${ }^{3}$, suas opções de isometria do plano o Tangram. A metodologia utilizada foi a revisão sistemática de literatura, e iniciou com buscas realizadas nos periódicos da Biblioteca Digital Brasileira de Teses e Dissertações (BDTD), no Banco de Teses e Dissertações da CAPES e do Google Acadêmico, sendo que, após análise baseada no protocolo estabelecido pela revisão sistemática, foram selecionados 10 trabalhos, sendo 7 dissertações e 3 artigos.

O software dinâmico GeoGebra, um dos focos do nosso estudo, é livre, de caráter matemático e foi desenvolvido por Markus Hohenwarter em sua tese de doutorado no ano de 2001 na Universidade de Salzburgo, Áustria. Markus o desenvolveu objetivando obter uma ferramenta adequada ao ensino de Matemática, conciliando entes algébricos aos geométricos (daí o nome: GeoGebra = Geometria e Álgebra). Esse software caracteriza-se por permitir várias representações para um só objeto matemático e por tornar possível a sua manipulação em tela. Dentre os seus diversos recursos o GeoGebra pode contribuir na dinamicidade e interatividade no ensino das isometrias do plano. Mas, afinal o que é isometria do plano mesmo?

Isometrias do plano, segundo Souza e Pataro (2014), são transformações geométricas que preservam as distâncias entre os pontos e as amplitudes dos ângulos e, assim, transformam uma figura em outra geometricamente congruente. Observa-se que no GeoGebra, conforme figura 1, as isometrias do plano são classificadas em quatro tipos: reflexão em relação a uma reta, reflexão em relação a um ponto, rotação em torno de um ponto e translação por um vetor.

\footnotetext{
${ }^{3} \mathrm{O}$ GeoGebra é um software de matemática dinâmica que permite a combinação de procedimentos geométricos e algébricos. Acessando o site - http://www.geogebra.org - pode-se instalar gratuitamente em qualquer computador.
} 
Figura 1.

Menu das isometrias no plano no GeoGebra

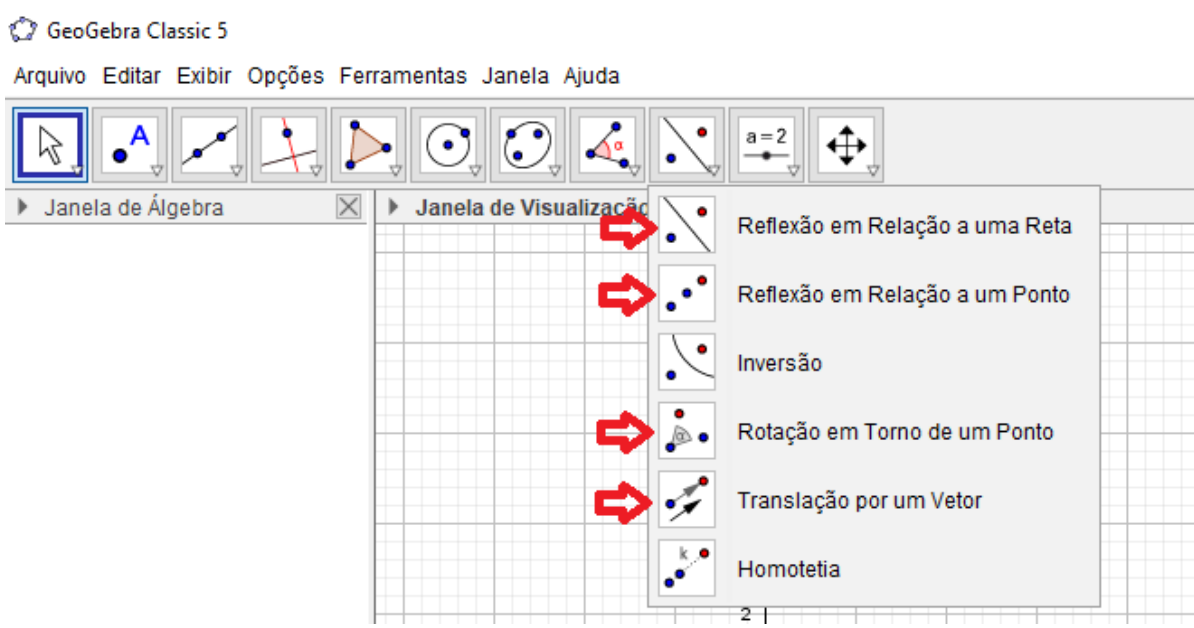

As outras duas opções vistas na figura não são isométricas, pois tanto a homotetia, quanto a inversão, mudam as dimensões das figuras após as transformações.

O terceiro elemento presente nessa revisão sistemática é o Tangram (figura 2), que, segundo Ribeiro et al. (2012), é um quebra-cabeça chinês, de origem milenar, com o qual podese formar cerca de 1700 figuras. Diversas lendas procuram explicar o seu surgimento. Contudo, independentemente de qual seja a história sobre a criação do Tangram, este quebra-cabeça tem sido, cada vez mais, utilizado como recurso didático nas aulas de Matemática, já que, segundo Souza et al (2008), ele apresenta um forte aspecto lúdico, que proporciona aos alunos o desenvolvimento de habilidades e competências no contexto da matemática de forma prazerosa.

Figura 2.

Representação do Tangram

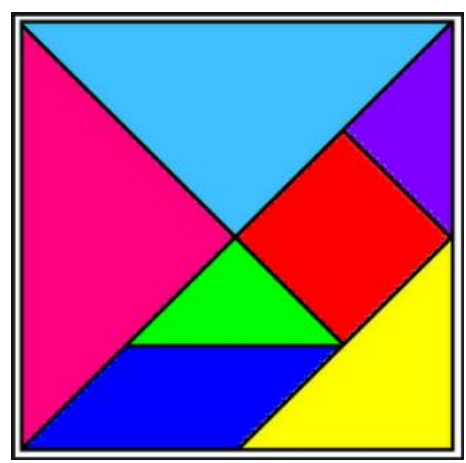


Segundo Fink (2005, p. 3) a melhor definição para revisão sistemática é: “o método sistemático, explícito, abrangente e reprodutível para identificar, avaliar e sintetizar o corpo existente de estudos completos produzidos por pesquisadores, estudiosos e profissionais". Ainda segundo o autor, uma revisão sistemática autônoma rigorosa se compromete a ser sistemática ao seguir uma abordagem metodológica; explícita na explicação dos procedimentos pelos quais foi construída; abrangente ao abordar de forma completa o material de relevância; e, de forma segura, reprodutível por outros pesquisadores que desejarem seguir tal abordagem.

De acordo com Mendes e Pereira (2020), é de suma importância que um guia com etapas para realizar revisões sistemáticas seja suficientemente claro, buscando orientar e facilitar o trabalho dos pesquisadores. Corroborando com esse raciocínio, seguimos as orientações de Kitchenham (2004), que conceitua uma revisão sistemática como um meio de identificar, avaliar e interpretar diversas produções de pesquisa disponível para uma ou mais determinada questão a que se propõe pesquisar ou para uma específica área de pesquisa. Para ele existem muitas razões para se realizar uma RS, entre as quais podemos destacar:

- Reunir evidências que dizem respeito a um determinado assunto ou tecnologia;

- Identificar lacunas na pesquisa atual;

- Fornecer embasamento para o posicionamento apropriado de novas atividades de pesquisa.

Geralmente, nas introduções e seções teóricas de trabalhos científicos são encontradas revisões sistemáticas. Ainda de acordo com Kitchenham (2004), para atingir os resultados, alguns pontos necessitam ser claramente definidos, normalmente em um instrumento conhecido como protocolo da revisão, que geralmente contém os seguintes itens: (1) o objetivo da pesquisa; (2) a questão da pesquisa; (3) os descritores de busca; (4) os critérios de inclusão e exclusão para estudos; (5) as bases de dados da pesquisa; (6) estratégia de análise. 
Esse artigo explicita os critérios de inclusão e exclusão que caracterizam o protocolo que realizará de forma sistemática as buscas e escolhas dos estudos selecionados. O protocolo utilizado nesta pesquisa inicialmente foi amparado pela definição do objetivo e pela questão de pesquisa que são: (1) objetivo da pesquisa: analisar dissertações e artigos que discutam a influência do software GeoGebra com suas opções de isometria do plano e a atividade lúdica do Tangram na melhoria dos processos de ensino e aprendizagem de matemática no ensino Básico. (2) Questão de pesquisa: quais as vantagens da utilização das opções de isometria do plano do software GeoGebra e do Tangram nos processos de ensino de matemática no ensino básico? Esses dois elementos viabilizaram as escolhas dos descritores de busca que vão nortear as investigações necessárias par a realização da presente pesquisa.

Para iniciar as buscas, nas já referidas bases de dados, foram utilizados os pares de descritores Tangram/Matemática, Tangram/GeoGebra e, por último, GeoGebra/Isometria. É de suma importância justificar que como não foram encontrados trabalhos que envolvessem os descritores Tangram, GeoGebra e opções de isometria de forma simultânea foi necessária a utilização desses descritores em pares, incluindo o descritor Matemática para se associar ao descritor Tangram no intuito de encontrar trabalhos que associassem esse elemento lúdico ao ensino de Matemática.

Objetivando elencar os resultados encontrados por intermédio do levantamento feito sobre o assunto, a partir dos trabalhos selecionados, descreveremos, por meio da análise dos dados, os quais, serão apresentados sucintamente em forma de tabelas e gráficos e logo em seguida discutidos, tais como: frequências desses elementos nas bases de dados; título e autores dos trabalhos; instituições de defesa dos estudos, seus referidos Estados e ano de conclusão; questões de pesquisa; abordagem metodológicas e técnicas e instrumentos utilizados na pesquisa e , por último, resultado do trabalho.

\section{Os critérios de inclusão e exclusão do protocolo}


No intuito de identificar apenas os artigos e dissertações que abordem significativamente a questão de pesquisa, foram definidos os critérios de inclusão de exclusão dos estudos. A organização dos critérios definidos neste estudo está representada conforme tabela 1.

Tabela 1.

Critérios de inclusão e exclusão

\begin{tabular}{l|l}
\hline \hline \multicolumn{1}{c}{ Inclusão } & \multicolumn{2}{c}{ Exclusão } \\
\hline \hline $\begin{array}{l}\text { Estudos relacionados ao uso do GeoGebra } \\
\text { e seus comandos de isometria e o Tangram } \\
\text { como proposta de beneficiar o processo } \\
\text { ensino aprendizagem de Matemática. }\end{array}$ & $\begin{array}{l}\text { Estudos escritos em idiomas que não } \\
\text { sejam o português. }\end{array}$ \\
\hline \hline Estudos publicados de 2015 até 2020. & Estudos com menos de 8 páginas. \\
\hline $\begin{array}{l}\text { Estudos que estejam acessíveis via web } \\
\text { gratuitamente. }\end{array}$ & Estudos duplicados. \\
\hline \hline $\begin{array}{l}\text { Estudos relacionados à conteúdos que } \\
\text { estejam na grade curricular do Ensino } \\
\text { Básico. }\end{array}$ & Estudos incompletos. \\
\hline \hline Estudos que possuem resumo. & \\
\hline \hline
\end{tabular}

\section{As bases de dados e os descritores de busca}

Nessa seção objetiva-se expor o levantamento dos estudos já realizados sobre a temática envolvendo GeoGebra, Isometria e Tangram no contexto da matemática. Foram consultadas as bases de dados CAPES, BDTD e Google Acadêmico, utilizando os descritores de busca Tangram/Matemática, Tangram/GeoGebra e GeoGebra/Isometria. Após a identificação dos estudos relacionados aos descritores de busca mencionados, bem como da aplicação dos critérios de inclusão e exclusão previamente definidos, selecionamos os estudos que melhor poderiam representar o nosso interesse de pesquisa.

Na figura 3 é apresentado um panorama geral do processo de filtragem dos estudos. No primeiro momento do processo de busca, utilizou-se os filtros determinados pelos critérios ano 
de publicação e idioma que são possíveis de serem indicados nos sites das bases de dados. É importante lembrar que as datas que determinaram as buscas tiveram como limite, o início do ano 2015, e término em 25 de setembro do ano 2020, quando foram finalizadas as buscas, sendo encontrados 293 títulos. Em seguida, foram realizados os filtros utilizando os critérios, conforme tabela 1, analisando-se os títulos dos estudos, que após serem analisadas as pertinências ao objetivo da pesquisa, foram selecionados 84 títulos. O penúltimo filtro foi aplicado, após leitura criteriosa dos resumos dos estudos, utilizando-se novamente os critérios (tabela 1), a partir dos quais, foram escolhidos 16 títulos. Para finalizar, foi realizada uma filtragem utilizando-se novamente critérios de inclusão e exclusão (tabela 1) e, após leitura completa dos 16 estudos selecionados, foram selecionados os 10 trabalhos que mais se identificavam com os objetivos da pesquisa.

Figura 3.

\section{Filtragem dos Estudos}

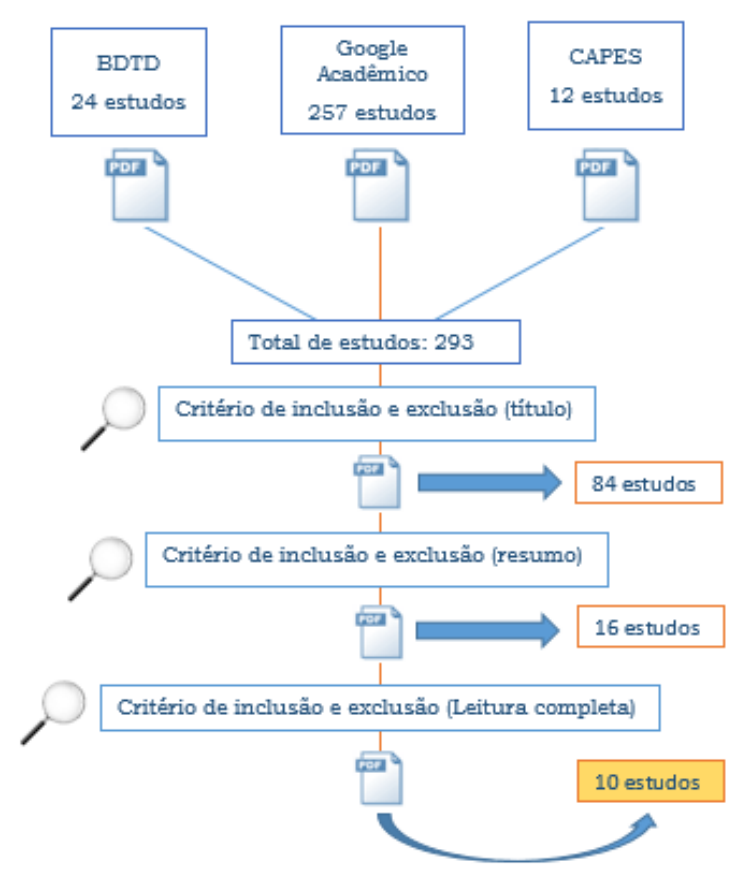

Na figura 4 é feita uma quantificação individualizada da filtragem referente a cada base usada como fonte de pesquisa. Percebe-se que houve uma predominância da base de dados da BDTD com o total de 05 pesquisas selecionadas, seguida do Google Acadêmico com 04 
pesquisas e, por último, a CAPES com 01 estudo selecionado, totalizando os 10 trabalhos que melhor poderiam qualificar a atual pesquisa.

Figura 4.

Quantificação da filtragem dos estudos

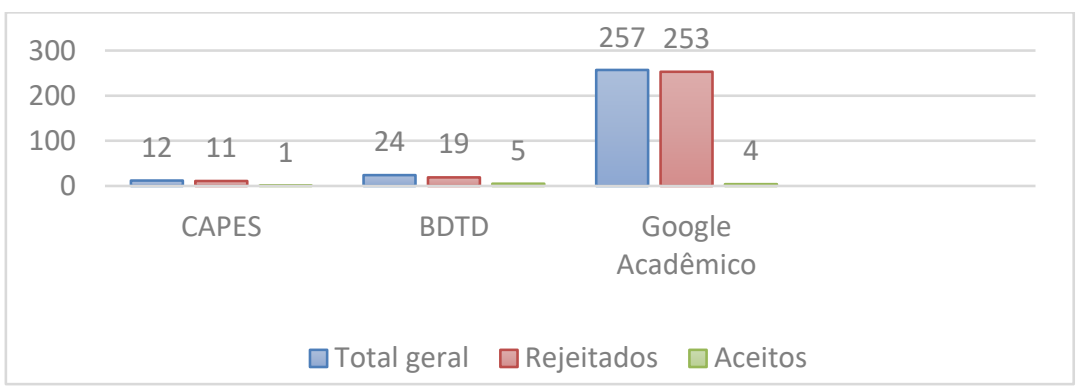

É importante salientar que quando uma mesma pesquisa pode ser encontrada em duas ou mais fontes, priorizou-se a CAPES e na sequência a BDTD.

\section{Os títulos e autores dos estudos}

Objetivando enumerar as informações encontradas por meio do levantamento realizado sobre a temática, no intuito de responder à questão de pesquisa, diferentes dados foram extraídos de cada estudo selecionado. Descrevemos seus aspectos mais relevantes em forma de tabelas e gráficos e, além disso, relacionaremos essas abordagens entre si e, nas considerações do atual artigo, faremos algumas inferências a respeito dos resultados dos trabalhos selecionados e sobre uma nova perspectiva para a utilização do GeoGebra, Tangram e isometrias do plano.

Pretendendo tornar mais fácil a identificação dos estudos, faremos a sua classificação numa sequência de A1 a A3, em que o "A" representa a palavra artigo e a sequência de D1 a D7, onde "D" representa o vocábulo dissertação, concordando com os títulos e os respectivos autores, conforme a tabela 2 . 
Tabela 2.

Título e autor (a) dos estudos encontrados

\begin{tabular}{|c|c|c|}
\hline $\begin{array}{l}\text { Abreviatura } \\
\text { do estudo }\end{array}$ & Título & Autor(a) \\
\hline D1 & $\begin{array}{l}\text { Uma sequência didática para o ensino de } \\
\text { transformações geométricas com } \\
\text { GeoGebra. }\end{array}$ & PIMENTEL, L.F.G \\
\hline D2 & $\begin{array}{l}\text { Estudo da isometria por meio do software } \\
\text { GeoGebra. }\end{array}$ & OLIVEIRA, E.M.G \\
\hline D3 & Isometrias no Ensino Básico. & BULGARELLI, C.C.B \\
\hline D4 & $\begin{array}{l}\text { GeoGebra e isometrias: a ação de arrastar na } \\
\text { construção de conceitos. }\end{array}$ & DICKEL, M.T \\
\hline D5 & $\begin{array}{l}\text { Investigando o ensino de geometria na } \\
\text { educação de jovens e adultos: um estudo de } \\
\text { caso com alunos e professores. }\end{array}$ & SANTOS, A.A \\
\hline D6 & $\begin{array}{l}\text { O uso do Tangram como proposta no ensino } \\
\text { de frações. }\end{array}$ & SANTOS, S.F \\
\hline D7 & $\begin{array}{l}\text { Tangram e resolução de problemas: desafios } \\
\text { e possibilidades. }\end{array}$ & COSTA, S. M \\
\hline A1 & $\begin{array}{l}\text { Incluindo tecnologias no currículo de } \\
\text { matemática: planejando aulas com o recurso } \\
\text { dos tablets. }\end{array}$ & $\begin{array}{l}\text { HOMA, A.I.R \& } \\
\text { GROENWALD, C.L.O }\end{array}$ \\
\hline $\mathrm{A} 2$ & $\begin{array}{l}\text { Uso do Tangram como material lúdico em } \\
\text { sala de aula. }\end{array}$ & $\begin{array}{l}\text { PONTES, D.F.N \& LOPES, } \\
\text { S.C.C }\end{array}$ \\
\hline A3 & $\begin{array}{l}\text { Práticas multidisciplinares: atividades lúdicas } \\
\text { e tecnologia digital aliada ao estudo de artes e } \\
\text { geometria. }\end{array}$ & $\begin{array}{l}\text { SILVA, R.S.S \& } \\
\text { GAUTÉRIO, V.L.B }\end{array}$ \\
\hline
\end{tabular}

Como já foi dito anteriormente, os descritores utilizados para a realização das buscas pelos estudos foram, GeoGebra/Tangram, GeoGebra/Isometria e Tangram/Matemática. Ao analisar os títulos é possível identificar que em 8 dos 10 estudos selecionados existe pelo menos um desses descritores. Vale ressaltar que, apesar da ausência desses descritores nos títulos dos estudos D5 e A3, o vocábulo geometria presente no título nos motivou a fazer uma leitura do 
resumo e, após isso, percebemos a relevância do trabalho para os nossos interesses de pesquisa. Inclusive, em nossas buscas encontramos alguns estudos que continham dois ou mais dos descritores estabelecidos e quando fizemos a leitura do resumo notamos que a referida pesquisa, por diversos motivos, não se enquadrava entre os nossos interesses de estudo previamente estabelecidos pelo protocolo de pesquisa, ás vezes, pela pesquisa não ter sido aplicada ao ensino básico, outras por estudo incompleto e até mesmo por não possuir um resumo do trabalho.

\section{As instituições de defesa dos estudos, seus referidos estados, regiões e ano de conclusão.}

A tabela 3, foi estruturada objetivando oferecer uma visão panorâmica das instituições, dentre elas, universidades, faculdades ou centros de pesquisa, seus referidos estados e ano em que foram concluídos os estudos da referida pesquisa.

\section{Tabela 3.}

Instituições de defesa dos estudos, seus referidos estados e ano de conclusão

\begin{tabular}{c|l|c|c|}
\hline \hline $\begin{array}{c}\text { Abreviatura } \\
\text { do estudo }\end{array}$ & \multicolumn{1}{|c}{ Instituição de defesa do estudo } & Estado & Ano \\
\hline D1 & Universidade Federal de São Carlos (UFSCAR) & SP & 2016 \\
\hline D2 & Universidade do Vale do Taquari (UNIVATES) & MA & 2018 \\
\hline D3 & Universidade Estadual de Campinas (UNICAMP) & SP & 2018 \\
\hline D4 & Universidade Federal do Rio Grande do Sul (UFRS), & RS & 2019 \\
\hline D5 & Universidade Federal de Goiás (UFG) & GO & 2018 \\
\hline D6 & Universidade Federal de Goiás (UFG), & GO & 2019 \\
\hline D7 & Universidade Estadual da Paraíba (UEPB) & PB & 2019 \\
\hline A1 & $\begin{array}{l}\text { UNIÓN - Revista Ibero Americana de Educação } \\
\text { Matemática }\end{array}$ & RS & 2016 \\
\hline A2 & Sociedade Brasileira de Educação Matemática (SBEM) & PA & 2016 \\
\hline
\end{tabular}


A3 Revista Latino-Americana de Estudos em Cultura e Sociedade (RELA Cult)

Percebe-se, observando a tabela 03, que a distribuição cronológica das conclusões dos trabalhos, cuja busca pertenceu ao intervalo de 2015 a 2020, concentrou-se nos anos de 2016, 2018 e 2019, sendo que nos anos de 2016 e 2018 foram concluídos 03 trabalhos em cada ano e em 2019 foram concluídos 04 trabalhos. Nota-se que, nos anos 2015, 2017 e 2020 não foi publicado nenhum dos estudos selecionados para embasar a nossa pesquisa.

Também na tabela 03, é constatado que o estado brasileiro que mais forneceu estudos para a nossa pesquisa foi o Rio Grande do Sul, com 03 trabalhos selecionados, seguido de São Paulo e Goiás com 02 estudos cada um e, em seguida, os estados do Maranhão, Pará e Paraíba com 01 estudo selecionado em cada um. Nota-se que, mesmo seguindo com rigor o protocolo da revisão sistemática, todas as regiões brasileiras tiveram pelo menos uma instituição que colaborou para a obtenção de dados necessários a realização da atual pesquisa. Dos 10 estudos selecionados somente 02 foram realizados por pesquisadores de uma mesma instituição, a Universidade Federal de Goiás (UFG). Os demais estudos foram realizados por pesquisadores vinculados a instituições distintas. Percebe-se que 7 dos 10 estudos selecionados foram produzidos por pesquisadores vinculados à Universidades estaduais ou federais, enquanto os outros três, foram distribuídos igualmente entre duas revistas e a Sociedade Brasileira de Educação Matemática (SBEM).

\section{As abordagens metodológicas e técnicas e instrumentos utilizados nas pesquisas}

$\mathrm{Na}$ medida em que o pesquisador determina a metodologia para o seu trabalho ele define a maneira pela qual realizará o seu estudo, tal como, a coleta das informações e encaminhamentos para análise dos resultados da sua pesquisa. Em sua grande maioria os autores discutem a respeito da natureza das metodologias nos trabalhos e as descrevem como sendo qualitativa, quantitativa e quali-quantitativa. Já o tipo de abordagem é classificado como: 
estudo de caso, pesquisa-ação, etnográfica, documental, exploratória, bibliográfica, colaborativa, interventiva, e outras mais. Em suas pesquisas os autores definem também quais os instrumentos e técnicas utilizados para a produção dos dados a serem analisados.

Na tabela 4 são descritas quais foram as abordagens metodológicas adotadas pelos autores dos estudos. Além disso, determina também quais os instrumentos e técnicas utilizadas. Em muitos casos esses elementos foram indicados no resumo do estudo, enquanto em outros casos foi necessária uma leitura da sua parte metodológica para identificação desses elementos.

Tabela 4.

Abordagem metodológicas e técnicas e instrumentos utilizados na pesquisa

\begin{tabular}{|c|c|c|}
\hline $\begin{array}{l}\text { Abreviatura } \\
\text { do estudo }\end{array}$ & Técnicas e instrumentos de pesquisa & $\begin{array}{l}\text { Abordagem } \\
\text { metodológica }\end{array}$ \\
\hline D1 & $\begin{array}{l}\text { Observação participante, entrevista, questionário e análise } \\
\text { documental das avaliações dos alunos. }\end{array}$ & $\begin{array}{l}\text { Qualitativa de } \\
\text { tipo interventiva }\end{array}$ \\
\hline D2 & $\begin{array}{l}\text { Foram realizados dois grupos de discussões, gravações de } \\
\text { áudio e vídeos, e anotações em diário de campo }\end{array}$ & $\begin{array}{l}\text { Qualitativa de } \\
\text { tipo interventiva }\end{array}$ \\
\hline D3 & $\begin{array}{l}\text { Observação participante, e análise das atividades } \\
\text { realizadas. }\end{array}$ & $\begin{array}{l}\text { Qualitativa de } \\
\text { tipo interventiva }\end{array}$ \\
\hline D4 & $\begin{array}{l}\text { Gravações de vídeo coletivo das atividades, registros } \\
\text { escritos feitos pelos alunos, observações da pesquisadora e } \\
\text { arquivos do GeoGebra produzidos pelos alunos. }\end{array}$ & $\begin{array}{l}\text { Qualitativa de } \\
\text { tipo } \\
\text { investigativa }\end{array}$ \\
\hline D5 & $\begin{array}{l}\text { Os dados foram coletados por intermédio destes } \\
\text { questionários, das atividades e observações, } \\
\text { acompanhados de uma análise quantitativa e qualitativa } \\
\text { dos resultados que foram apresentados na forma de } \\
\text { gráficos e tabelas. }\end{array}$ & $\begin{array}{l}\text { Qualitativa de } \\
\text { tipo estudo de } \\
\text { caso }\end{array}$ \\
\hline D6 & $\begin{array}{l}\text { Levantamento de dados em livros, dissertações, teses e } \\
\text { artigos científicos que possuem como foco na utilização } \\
\text { do Tangram no contexto de frações. }\end{array}$ & $\begin{array}{l}\text { Qualitativa de } \\
\text { tipo } \\
\text { bibliográfico }\end{array}$ \\
\hline D7 & Análise de livros didáticos por categorias de sentido. & $\begin{array}{l}\text { Qualitativa de } \\
\text { tipo } \\
\text { bibliográfico }\end{array}$ \\
\hline
\end{tabular}




\begin{tabular}{|l|l|l|}
\hline A1 & $\begin{array}{l}\text { Observações realizadas nos dois grupos de trabalho, no } \\
\text { replanejamento das atividades propostas ao grupo de } \\
\text { professores incorporando suas sugestões e reflexões sobre } \\
\text { o uso das mesmas. }\end{array}$ & $\begin{array}{c}\text { Qualitativa de } \\
\text { tipo interventiva }\end{array}$ \\
\hline A2 & $\begin{array}{l}\text { Observação participante, breve entrevista e análise do } \\
\text { desempenho dos alunos diante das atividades de uma } \\
\text { sequência didática. }\end{array}$ & $\begin{array}{c}\text { Qualitativa de } \\
\text { tipo interventiva }\end{array}$ \\
\hline A3 & $\begin{array}{l}\text { Observação participada durante a realização da construção } \\
\text { do Tangram com dobradura e através de exploração do } \\
\text { software GeoGebra. }\end{array}$ & $\begin{array}{c}\text { Qualitativa de } \\
\text { tipo interventiva }\end{array}$ \\
\hline
\end{tabular}

A informações da tabela 4 permitem compreender que todas as dissertações analisadas apresentaram a abordagem metodológica no modelo qualitativo, diferenciando-as apenas nos tipos de pesquisas. Percebe-se também que houve uma predominância do tipo pesquisa interventiva, pois tivemos 6 estudos desse tipo como preferências dos autores entre os 10 estudos selecionados. A segunda mais adotada foi a do tipo bibliográfica e, observa-se também, que as pesquisas do tipo estudo de caso e investigativa foram escolhidas uma vez cada. Em relação às técnicas e instrumentos de pesquisa, a tabela 04 evidencia que os autores utilizaram mais de uma técnica ou instrumento para a produção dos dados. Percebe-se também que não existiu uma relação entre a metodologia aplicada e os instrumentos e técnicas para produção de dados, pois, mesmo os autores escolhendo a mesma metodologia, conforme informações da tabela 4, as técnicas e instrumentos utilizados foram bem diferentes. De forma geral, os autores utilizaram uma diversidade de técnicas e instrumentos, com destaque para: questionários, entrevistas, testes e provas, observações participadas, gravações em vídeo, diário de campo, software, livros, dissertações, teses e artigos científicos.

\section{As questões de pesquisa}

Segundo Gomides (2002), um problema de pesquisa consiste em expressar de maneira explícita, clara, compreensível e operacional, qual a dificuldade que está exposta para o pesquisador, a qual, ele pretende resolver. O autor diz ainda que o objetivo da elaboração do 
problema da pesquisa é tornar específica a meta a ser alcançada. Uma boa parte dos pesquisadores, cujas obras foram selecionadas, não relataram as questões de pesquisa em seus respectivos resumos. Nesses casos, foi necessária uma leitura das suas considerações e, às vezes, até uma leitura geral dos trabalhos para poder identificar as suas questões de pesquisa. Como alguns autores, nem mesmo em toda a obra, explicitaram as questões em forma de pergunta, tivemos que considerar, conforme tabela 5 , os seus objetivos gerais como dificuldade a ser superada.

Tabela 5.

Questão de pesquisa

\begin{tabular}{|c|c|}
\hline $\begin{array}{l}\text { Abreviatura } \\
\text { do estudo }\end{array}$ & Questão de pesquisa \\
\hline D1 & $\begin{array}{l}\text { Averiguar em que medida o GeoGebra promove uma melhora na situação de } \\
\text { ensino-aprendizagem, rompendo com o fracasso no ensino das transformações } \\
\text { isométricas. }\end{array}$ \\
\hline D2 & $\begin{array}{l}\text { Desenvolver uma proposta de formação continuada junto aos professores de } \\
\text { Matemática de uma escola da rede pública de Amarante do Maranhão usando } \\
\text { os recursos tecnológicos, , objetivando um estudo da isometria por meio do } \\
\text { software GeoGebra. }\end{array}$ \\
\hline D3 & $\begin{array}{l}\text { Construir um caleidoscópio no GeoGebra, trabalhando os conceitos de } \\
\text { simetria de reflexão e rotação e proporcionar aos alunos um contato com as } \\
\text { ferramentas do software através de uma atividade lúdica. }\end{array}$ \\
\hline D4 & $\begin{array}{l}\text { Como o recurso de arrastar no ambiente de geometria dinâmica do GeoGebra } \\
\text { contribui para a construção de conceitos de isometrias por meio do trabalho } \\
\text { com imagens de ilusão de óptica? }\end{array}$ \\
\hline D5 & $\begin{array}{l}\text { Que fatores podem ser identificados, que influenciam no ensino de Geometria } \\
\text { na Educação de Jovens e Adultos quanto às dificuldades enfrentadas pelos } \\
\text { docentes, aos recursos didáticos e à receptividade dos alunos? }\end{array}$ \\
\hline D6 & $\begin{array}{l}\text { Realizar um estudo teórico acerca dos conceitos de frações, bem como } \\
\text { verificar as potencialidades do uso do Tangram para o ensino deste conceito. }\end{array}$ \\
\hline D7 & $\begin{array}{l}\text { Refletir sobre os desafios e possibilidades do uso do Tangram associado à } \\
\text { resolução de problemas. }\end{array}$ \\
\hline
\end{tabular}




\begin{tabular}{|c|l|}
\hline A1 & $\begin{array}{l}\text { Investigar possibilidades do uso do Tangram em tablets como um recurso } \\
\text { didático na construção do conhecimento matemático. }\end{array}$ \\
\hline A2 & $\begin{array}{l}\text { Apresentar a importância do Tangram como jogo lúdico, e ainda mostrá-lo } \\
\text { como ferramenta facilitadora no processo de ensino-aprendizagem. }\end{array}$ \\
\hline A3 & $\begin{array}{l}\text { Discutir, refletir e ressignificar, conceitos geométricos a partir de práticas } \\
\text { multidisciplinares através de atividades lúdicas auxiliadas pelas tecnologias } \\
\text { digitais como o Tangram. }\end{array}$ \\
\hline
\end{tabular}

A análise da tabela 05 nos permite observar que os autores diversificaram bastante em relação aos interesses de pesquisa. As quatro primeiras obras, de D1 até D4, se debruçaram nos estudos envolvendo o uso do software GeoGebra vinculado as opções de isometria, ou algumas delas, que, como já dissemos, são a reflexão de uma figura em relação a uma reta e em relação a um ponto, a rotação de uma figura em relação a um determinado ponto referencial e a translação de uma figura por um vetor dado. No estudo D1, Pimentel (2016) propõe averiguar em que medida o software dinâmico Geogebra pode promover uma melhora na situação de ensino-aprendizagem, rompendo, dessa forma, com o insucesso no ensino das transformações isométricas. No estudo D2, Oliveira (2018) objetiva desenvolver uma proposta de formação continuada junto aos professores de Matemática de uma escola da rede pública de Amarante do Maranhão, utilizando os recursos tecnológicos, especificamente objetivando um estudo da isometria por meio do software dinâmico GeoGebra. Na pesquisa D3, Bulgarelli (2018) propõe aos alunos construir um caleidoscópio no GeoGebra, trabalhando os conceitos de simetria de reflexão e rotação (nota-se que não utilizou a rotação por um determinado ponto), proporcionando aos alunos um contato com as ferramentas do software através de uma atividade lúdica. Finalizando esses quatro estudos, na pesquisa D4, Dickel (2019) questiona de que forma o recurso de arrastar no ambiente do GeoGebra contribui para a formação de conceitos de isometrias por meio da atividade com figuras de ilusão de óptica.

Do estudo D5 até o estudo D7, os autores abordaram a utilização do Tangram como potencializador do estudo de matemática. Na dissertação D5, Santos (2018) realiza uma 
investigação a respeito do ensino de geometria na educação de jovens e adultos (EJA), questionando especificamente sobre quais fatores podem ser identificados, que influenciam no ensino de Geometria na EJA quanto às dificuldades enfrentadas pelos docentes, aos recursos didáticos e à receptividade dos discentes. Nessa pesquisa o autor utiliza o Tangram como facilitador do ensino de geometria. No estudo D6, Santos (2019), propõe um estudo teórico abordando os conceitos de frações, intencionando verificar as potencialidades do uso do Tangram para o aprendizado dos conceitos relacionados a esse estudo. Na dissertação D7, Costa (2019), direciona seus estudos a respeito de uma reflexão sobre os desafios e possibilidades da utilização do Tangram à resolução de problemas. Essa reflexão foi possibilitada através de uma análise de livros didáticos por categorias de sentido e das entrevistas com professores dos diversos segmentos da educação.

Finalmente, nos trabalhos A1, A2 e A3, foram abordadas as pesquisas que relacionavam o software GeoGebra e o elemento lúdico Tangram. É importante salientar que não encontramos dissertações e nem teses nas as bases de dados CAPES, BDTD e Google Acadêmico, que estudassem simultaneamente esses dois elementos. Como já foi mencionado anteriormente, esses três estudos foram encontrados na categoria de artigos. No artigo A1, Homa e Groenwald (2016), realizaram a pesquisa com o intuito de investigar as possibilidades do uso do Tangram em tablets, utilizando o software GeoGebra, como um recurso didático na construção do conhecimento matemático. Esse trabalho abordou conceitos de geometria e coordenadas polares. No artigo A2, Pontes e Lopes (2016), esclarecem a importância do Tangram como jogo lúdico e, em parceria com o GeoGebra, evidenciam sua utilidade como ferramenta facilitadora no processo de ensino-aprendizagem de geometria. Finalizando as nossas observações a respeito das questões de pesquisa dos estudos selecionados protocolarmente, temos o artigo A3, no qual, Silva e Gautério (2019), ressignificam os 
conceitos geométricos a partir de práticas multidisciplinares através de atividades lúdicas auxiliadas pelas tecnologias digitais como o GeoGebra combinadas ao ludismo do Tangram.

De forma geral, ao analisar as questões de pesquisa que motivaram os estudos definidos pelas duplas de descritores formados pela tríade GeoGebra/Isometrias/Tangram percebemos que essas questões normalmente se referiam as vantagens e desafios da utilização das Tecnologias Digitais da Informação e Comunicação (TDIC) no processo de ensino e aprendizagem de Matemática, quando mencionavam às opções de isometria do plano do Geogebra. Já quando se trata de incluir a utilização do Tangram, as questões tendem a sugerir investigações a respeito da importância do Tangram como alternativa lúdica e ainda estudá-lo como provável ferramenta facilitadora no processo de ensino-aprendizagem de Matemática.

\section{Os principais resultados das pesquisas}

Em toda pesquisa, existe um resultado. Depois de realizar uma investigação, o pesquisador deverá expressar os frutos de sua pesquisa, quais foram os resultados oriundos do seu estudo, a conclusão de sua investigação. Nesta revisão sistemática, da mesma forma que foi necessária uma observação mais apurada para identificar as questões de pesquisa, já que elas não estavam mencionadas no resumo, para analisar os resultados das pesquisas apresentadas também, foi preciso, em alguns estudos, realizar uma leitura das conclusões e/ou considerações das pesquisas. A tabela 6 auxiliará, mesmo sendo apenas um recorte dos pontos centrais dos resultados dos estudos, na compreensão dos resultados encontrados nas pesquisas selecionadas nesta revisão sistemática. 


\section{Tabela 6.}

\section{Resultados das pesquisas}

\begin{tabular}{|c|c|}
\hline $\begin{array}{l}\text { Abreviatura } \\
\text { do estudo }\end{array}$ & Resultados das pesquisas \\
\hline D1 & $\begin{array}{l}\text { Os resultados indicam que as TIC's contribuem de forma significativa no processo de ensino e } \\
\text { aprendizagem, contudo alguns fatores externos ainda são entraves para a efetiva } \\
\text { implementação deste recurso nas escolas. }\end{array}$ \\
\hline D2 & $\begin{array}{l}\text { Os resultados apontaram que: a) o software GeoGebra tem o potencial de auxiliar no ensino de } \\
\text { Matemática; b) o estudo abasteceu os professores de confiança para desenvolverem essa } \\
\text { tecnologia junto aos seus alunos; e c) o estudo também incentivou os professores a superarem } \\
\text { as práticas usadas tradicionalmente e aponta para mudanças futuras das posturas docentes dos } \\
\text { educadores. }\end{array}$ \\
\hline D3 & $\begin{array}{l}\text { Observamos que, através de atividades lúdicas o interesse dos alunos pelo tema é despertado e } \\
\text { eles se apresentam mais motivados para resolver problemas e exercícios que envolvam esse } \\
\text { conteúdo. Além disso, através da apresentação das questões da OBMEP os alunos passam a } \\
\text { enxergá-la de uma maneira diferente, desmistificada e se sentem mais motivados a participar. }\end{array}$ \\
\hline D4 & $\begin{array}{l}\text { Os resultados apontam para a compreensão de conceitos que envolvem as isometrias, que } \\
\text { surgem a partir da manipulação no software, em forma de arrastamento, conduzindo à } \\
\text { construção de pensamentos matemáticos em conjunto com a tecnologia. }\end{array}$ \\
\hline D5 & $\begin{array}{l}\text { Os alunos estudaram o conteúdo de uma forma alternativa, adquiriram uma visão mais ampla } \\
\text { de suas realidades sociais e econômicas, tiveram a oportunidade de trabalharem com temas } \\
\text { transversais e associarem o conteúdo estudado a outras áreas de conhecimento, }\end{array}$ \\
\hline D6 & $\begin{array}{l}\text { As tarefas realizadas com o auxílio de materiais manipulativos, especificamente o Tangram, } \\
\text { instigaram a curiosidade e contribuiu para o desenvolvimento do pensamento fracionário, a } \\
\text { abstração, a criatividade, a percepção espacial e a concentração do aluno. }\end{array}$ \\
\hline D7 & $\begin{array}{l}\text { Após a apreciação dos dados, constatou-se que os professores reconhecem as possibilidades do } \\
\text { uso do Tangram e que, associado à resolução de problemas, a utilização do Tangram pode } \\
\text { favorecer a motivação dos alunos, a compreensão de conceitos matemáticos, o } \\
\text { desenvolvimento da autonomia e da criatividade. }\end{array}$ \\
\hline A1 & $\begin{array}{l}\text { Os resultados apontam que o uso de tablets é uma alternativa metodológica para a inserção das } \\
\text { TIC na Educação Matemática, mudando o planejamento usual. }\end{array}$ \\
\hline A2 & $\begin{array}{l}\text { Proporcionou uma forma diversificada tanto em ensinar como aprender um campo da } \\
\text { matemática muito rico. Além de trabalhar geometria, utilizamos métodos de abranger } \\
\text { construções geométricas e o uso do GeoGebra. }\end{array}$ \\
\hline A3 & $\begin{array}{l}\text { A experiência mostrou que quando os estudantes são desafiados a operar com os conceitos, os } \\
\text { compreendem mais facilmente e as atividades lúdicas desenvolvem a autonomia, a criticidade, } \\
\text { a cooperação e colaboração, levam os estudantes a estabelecer relações entre o que já sabem e } \\
\text { o que desejam conhecer. }\end{array}$ \\
\hline
\end{tabular}

Com já foi dito antes, os quatro primeiros estudos, ou seja, de D1 até D4, se debruçaram aos estudos sobre as opções de isometria do plano vinculadas ao uso do software GeoGebra. 
Na dissertação D1, Pimentel (2016) idealizou e aplicou uma sequência didática com o uso do software GeoGebra envolvendo o tópico isometrias do plano, exceto translação por um vetor, em uma turma do $6^{\circ}$ ano do Ensino Fundamental. A sequência didática foi estruturada obedecendo as perspectivas da Engenharia Didática. Os resultados encontrados pelo autor indicaram que as Tecnologias da Informação e Comunicação (TIC's) contribuem de forma significativa no processo de ensino e aprendizagem. Porém, concluíram também que alguns fatores externos persistem como entraves para efetiva implementação destes recursos nas escolas. Na dissertação D2, Oliveira (2018) depois de uma análise a partir dos pressupostos da análise descritiva, os resultados apontaram que: a) o software GeoGebra tem potencial significativo no auxílio ao ensino de Geometria; b) o estudo incrementou corpo docente confiança no intuito de desenvolver essa tecnologia junto aso seus discentes; e c) o estudo incentivou o corpo docente a superar as práticas tradicionais de ensino da Geometria. Ainda segundo Oliveira, após o estudo, foi facilmente percebida a certeza da satisfação dos professores ao terem o acesso a fontes de conhecimento inovadoras, visando a melhoria de suas práticas pedagógicas. No estudo D3, Bulgarelli (2018) observou que, através de atividade lúdica envolvendo a construção de um caleidoscópio no GeoGebra, é despertado o interesse dos alunos pelo estudo dos comandos de isometrias e, também, demonstram maior motivação para solucionar os problemas e exercícios que abrangem tal conteúdo. Perceberam também que através da análise das questões da OBMEP, que foi um dos seus focos, os alunos passam a considerá-las de maneira mais significativa e mais dispostos a resolvê-las. Finalizando os quatro estudos que envolvem o GeoGebra e opções de isometria do plano, no estudo D4, Dickel (2019) encontrou bons resultados nas atividades geométricas resolvidas por estudantes do $3^{\circ}$ ano do Ensino Médio, com base na Teoria das Tecnologias Cognitivas e nas análises cognitivas do arrastar. Os resultados desse estudo apontam para a compreensão dos conceitos envolvendo isometria que surgem a partir da manipulação do GeoGebra, em forma de arrastamento, 
conduzindo à construção do pensamento matemático aliado à tecnologia. Em concordância com esses resultados temos Villa e Ruiz (2010) que perceberam a importância do software GeoGebra para estudar, estabelecer e demonstrar novas conjecturas de alguns conceitos matemáticos.

Só para lembrar, do estudo D5 até o estudo D7, os autores têm como tema a utilização do Tangram como facilitador do estudo de matemática. Na dissertação D5, Santos (2018) após a investigação, constatou que existe uma deficiência no ensino de geometria na EJA, e de acordo com depoimento dos discentes a implementação do projeto de intervenção foi válida e muito proveitosa na assimilação dos conteúdos, já que estudaram o conteúdo de uma forma alternativa. Além disso, adquiriram uma visão mais ampla de suas realidades sociais e econômicas, já que tiveram a oportunidade de trabalhar com temas transversais e associarem o conteúdo estudado a outras áreas de conhecimento. Na dissertação D6, Santos (2019) concluiu que o Tangram, quando utilizado adequadamente pelo professor, pode contribuir bastante para a formalização e apropriação de conceitos fracionários. Além disso, concluiu também que a utilização de recursos manipulativos, especificamente o Tangram, pode instigar a curiosidade, a abstração, a criatividade, a percepção espacial e a concentração dos alunos. No estudo D7, Costa (2019) constatou que a oficina ajudou na aliança da teoria com a prática a partir da utilização do Tangram como auxílio na resolução, exploração e proposição de problemas. Após a análise dos dados, foi constatado que os professores perceberam as diversas alternativas para o uso do Tangram e que, associado à resolução de problemas, a utilização dessa alternativa lúdica pode contribuir com a motivação discente, a melhor compreensão de conceitos, o desencadeamento da autonomia e da criatividade. Foram apontadas como dificuldades: a necessidade de criação de objetivos definidos e de uma metodologia adequada nas atividades; o desafio na proposição de problemas pelos discentes; a formação inadequada dos professores 
como empecilho na realização de experiências exitosas envolvendo resolução de problemas tendo o Tangram como potencializador da aprendizagem.

Nos artigos A1, A2 e A3, foram abordados os estudos que relacionavam o software GeoGebra e o Tangram. Novamente, salienta-se que não foram encontradas dissertações e nem teses nas as bases de dados CAPES, BDTD e Google Acadêmico, que abordassem simultaneamente o GeoGebra e o Tangram. No artigo A1, Homa e Groenwald (2016), após a finalização da pesquisa, concluíram que o uso dos objetos digitais sobre o plano quadriculado do GeoGebra permitiu a visualização das relações entre as áreas dos objetos e a unidade de área. $\mathrm{Na}$ construção dos objetos pelos alunos sobre o plano quadriculado, permitiu determinar as dimensões das arestas e os ângulos somente pela visualização das figuras geométricas oriundas das peças do Tangram, permitindo que o aluno trabalhasse com a representação polar sem a apresentação formal dos conceitos. Os resultados sugerem que atividades utilizando tablets é uma alternativa metodológica para a inclusão das TIC na Educação Básica.

No artigo A2, Pontes e Lopes (2016), após o término do trabalho, concluíram que foi proporcionada uma forma diversificada, tanto em ensinar, como aprender conceitos em um campo da matemática tão rico que é a geometria. A pesquisa apontou, através de breve entrevista com os alunos, que houve uma motivação pela busca do conhecimento e o gosto e envolvimento pela geometria plana. Notou-se também que, apesar da atividade não ter um caráter avaliativo, o Tangram teve como característica prender a atenção dos alunos na execução da sequência didática. Finalizando as observações a respeito das conclusões das pesquisas selecionadas, tem-se o artigo A3, no qual, Silva e Gautério (2019) concluem que experiência apontou que quando os alunos são desafiados a operar com os conceitos, eles os compreendem com maior facilidade e, além disso, as atividades lúdicas em grupo, no caso específico, o GeoGebra aliado ao Tangram, contribuem para o desenvolvimento da autonomia, 
da criticidade e da colaboração, pois os induzem a estabelecer relações entre os que já dominam o conteúdo e os que desejam aprender.

Podemos inferir a partir da análise dos resultados desses estudos a importância do GeoGebra como um intensificador do ensino da matemática tanto no aspecto da geometria, quanto da álgebra e como fomentador do interesse discente e docente pelo estudo das opções de isometria do plano. No que se refere ao Tangram identificamos a sua importância como propulsor da apropriação dos conceitos geométricos, instigador da curiosidade, além da sua significativa contribuição em relação à percepção espacial. Nas pesquisas que foram realizadas em grupos ficou explícita a contribuição do GeoGebra aliado ao Tangram como determinantes para o desenvolvimento da autonomia, da criticidade, da colaboratividade e da presença do prazer, que é inerente em atividades lúdicas, por parte dos alunos no processo ensinoaprendizagem de Matemática.

\section{Considerações}

A atual revisão sistemática de literatura pautou-se em uma análise com objetivo de desencadear reflexões acerca da temática da utilização do Tangram, GeoGebra e opções de isometria do plano no ensino da matemática, bem como identificar as características dos estudos desenvolvidos sobre esta temática, as ferramentas, os recursos, o período, as instituições vinculadas aos autores e as metodologias utilizadas para o desenvolvimento desse tipo de pesquisa, suas questões de pesquisa e os resultados encontrados. A partir das buscas realizadas nos portais de periódicos de teses e dissertação da Biblioteca Digital Brasileira de Teses e Dissertações (BDTD), no Banco de Teses e Dissertações da CAPES e no Google Acadêmico.

Usando como referência os resultados encontrados nesta pesquisa podemos inferir, dentre outras coisas, a respeito da significativa contribuição do GeoGebra como um potencializador do ensino da matemática e como incentivador do interesse discente pelo estudo 
das opções de isometria do plano; a contribuição do Tangram como facilitador da apropriação dos conceitos geométricos, instigador da curiosidade, propulsor da criatividade e mediador da percepção espacial; a contribuição das atividades lúdicas, em grupo, que utilizam o GeoGebra aliado ao Tangram para o desenvolvimento da autonomia, da criticidade, da sociabilidade e da colaboratividade entre alunos. Esses aspectos corroboram com o objetivo da nossa proposta de pesquisa de mestrado, pois acreditamos que proporcionar aos estudantes um ambiente motivador, utilizando recursos didáticos vinculados a elementos tecnológicos e lúdicos, impulsiona o estudante a ser agente propulsor da construção de seu próprio conhecimento. Sendo assim, constatamos que as contribuições proporcionadas por essa investigação nos permitiram vislumbrar e certificar as vantagens da utilização da tríade Tangram/Isometria do plano/GeoGebra no ensino da Matemática.

Por meio dessa revisão sistemática percebemos que o nosso objeto de estudo da nossa pesquisa é campo fértil para estudos, pois embora tenhamos encontrados diversos trabalhos com as mais variadas possiblidades de abordagem e proporcionando, assim, a percepção das muitas vantagens da utilização dos elementos citados no auxílio do ensino da matemática, notamos a ausência de estudos que envolvessem os três elementos de forma simultânea, ou seja, não encontramos nenhum estudo que abordassem o Tangram, o GeoGebra e as opções de isometria do plano como potencializadores de ensino da matemática. Faz-se necessário salientar que encontramos em Silva et al (2018) e Silva $(2020)^{4}$ algumas dessas construções, mas sem uma abordagem teórico-metodológica. É nessa nova perspectiva, a partir dessa lacuna, que estamos organizando nossa pesquisa, ou seja, com o aporte teórico-metodológico da Teoria da Atividade de Engestrom, queremos investigar as estratégias matemáticas dos alunos envolvidos nessas construções isometrias, durante a formação das figuras do Tangram no ambiente do GeoGebra. Assim, pretendemos realizar essa pesquisa com um grupo de alunos

\footnotetext{
${ }^{4}$ GeoGebra Materiais: https://www.geogebra.org/m/apMUMZ7M (acessado em: 24/09/2020).
} 
do $2^{\circ}$ ano do ensino médio de uma escola pública estadual, no Município de Vitória da Conquista (BA). Acreditamos que esse tipo de abordagem nos permitirá confirmar que essas construções são repletas de decisões e ações que envolvem diversos conceitos matemáticos, fazendo com que a atividade lúdica com o Tangram deixe de ser essencialmente trivial, se enveredando em um novo contexto, no qual, é facilmente identificada a necessidades de estratégias cognitivas mais exigentes.

Esperamos ao final de nossa investigação que, assim como, para nós, serviu de âncora para embasar nossos estudos, traga, também, contribuições a outros leitores interessados nesse campo de pesquisa.

\section{Referências}

Bulgarelli, C. C. de. (2018). Isometrias no Ensino Básico. [Dissertação apresentada ao Instituto de Matemática, Estatística e Computação Científica como parte dos requisitos exigidos para a obtenção do título de Mestrado Profissional - Universidade Estadual de Campinas, Campinas]. http://repositorio.unicamp.br/handle/REPOSIP/332216

Costa, S. M. da. (2019) Tangram e resolução de problemas: Desafios e possibilidades. [Dissertação de Mestrado pelo Programa de Pós-Graduação em Ensino de Ciências e Educação Matemática - PPGECEM, Universidade Estadual da Paraíba, Campina Grande]. https://bdtd.ibict.br/vufind/Record/UEPB_c0987b66fd2f5dccf59f6a4afae8fbf0

Dickel, M. T. (2019). GeoGebra e isometrias: a ação de arrastar na construção de conceitos. [Dissertação de Mestrado em Ensino de Matemática pelo Programa de Pós-Graduação em Ensino de Matemática, Universidade Federal do Rio Grande do Sul, Porto Alegre]

Da Silva, R. S. et al (2019). Práticas Multidisciplinares: Atividades Lúdicas e Tecnologia Digital aliada ao estudo de Artes e Geometria. RELACult-Revista Latino-Americana de Estudos em Cultura e http://periodicos.claec.org/index.php/relacult/article/view/1253

Fink, A. (2005). Conducting research literature reviews: From the Internet to paper. Thousand Oaks. https://www.worldcat.org/title/conducting-research-literature-reviews-from-theinternet-to-paper/oclc/55947868

Gomides, J. E. (2002). A definição do problema de pesquisa a chave para o sucesso do projeto de pesquisa. Revista do Centro de Ensino Superior de Catalão-CESUC-Ano IV. http://wwwp.fc.unesp.br/ verinha/ADEFINICAODOPROBLEMA.pdf

Homa, A. I. R., \& Groenwald, C. L. O. (2016). Incluindo tecnologias no currículo de matemática: planejando aulas com o recurso dos tablets. Revista Union. http://funes.uniandes.edu.co/17080/ 
Kitchenham, B. (2004). Procedures for performing systematic reviews, Technical Report TR/SE-0401. Department of Computer Science, Keele University and National ICT. Australia.

Mendes, L. O. R., \& Pereira, A. L. (2020). Revisão sistemática na área de Ensino e Educação Matemática: análise do processo e proposição de etapas. Educação Matemática Pesquisa, 22(3).

Oliveira, E. M. G. (2018). Estudo da isometria por meio do software GeoGebra: implicações pedagógicas de um curso de formação continuada com professores do $6^{\circ}$ ao $9^{\circ}$ ano em uma escola da rede pública de Amarante do Maranhão/MA. [Dissertação apresentada ao Programa de Pós-Graduação, Mestrado Profissional em Ensino de Ciências Exatas, da Universidade do Vale do Taquari - UNIVATES, Lajeado, Maranhão]. https://www.univates.br/bdu/handle/10737/2190

Pimentel, L. F. G. (2016). Uma sequência didática para o ensino de transformações geométricas com o GeoGebra. [Dissertação de Mestrado Profissional em Matemática vinculado ao Centro de Ciências Exatas e de Tecnologia da Universidade Federal de São Carlos (UFSCAR), São Carlos, São Paulo]. https://repositorio.ufscar.br/handle/ufscar/8267

Pontes, D. F. N, \& Lopes, S. C. (2016). Uso do Tangram como Material Lúdico em Sala de Aula. Sociedade Brasileira de Educação Matemática (SBEM), https://scholar.google.com.br/scholar?hl=pt-

BR\&as_sdt=0\%2C5\&q=USO+DO+TANGRAM+COMO+MATERIAL+L\%C3\%9A $\mathrm{DICO}+\mathrm{EM}+\mathrm{SALA}+\mathrm{DE}+\mathrm{AULA} \& \mathrm{btnG}=$

Ribeiro, E. M. P. et al. (2012). Sequência didática: Tangram. Sombrio. https://www.even3.com.br/anais/pibidsul/21261-o-tangram-como-metodologia-deensino-na-construcao-de-conceitos-matematicos/

Santos, A. A. de. (2018). Investigando o ensino de geometria na educação de jovens e adultos: um estudo de caso com alunos e professores. [Dissertação de Mestrado em Matemática em Rede Nacional, Universidade Federal de Goiás, Catalão]. https://repositorio.bc.ufg.br/tede/handle/tede/8606

Santos, S. F. dos. (2019). O uso do Tangram como proposta no ensino de frações. [ Dissertação de Mestrado em Matemática em Rede Nacional, Universidade Federal de Goiás, Jataí]. https://bdtd.ibict.br/vufind/Record/UFG_367b68b3c5c1f5be83e3a61c4ce2a81c

Silva, M. D. F et. al. (2018). Atividades Matemáticas com o GeoGebra. Amazon. https://www.amazon.com.br/kindledbs/hz/subscribe/ku?passThroughAsin=B07GK1 WFL3\&_encoding=UTF8\&shoppingPortalEnabled=true

Silva, M. D. F. (2020). GeoGebra. https://www.geogebra.org/m/apMUMZ7M

Souza, J.; Pataro, P. M. (2014). Vontade de saber matemática. São Paulo: Editora FTD.

Souza, E. R. de et al (2008). A Matemática das sete peças do Tangram. 1. ed. São Paulo-SP

Villa-Ochoa, J., \& Ruiz, M. (2010). Pensamiento variacional: seres-humanos-con-GeoGebra en la visualización de nociones variacionales. Educação Matemática Pesquisa, 12(3), 514-528.

Recebido em: 09/02/2021

Aprovado em: 24/03/2021 